\title{
Astragaloside IV Regulates the PI3K/Akt/HO-1 Signaling Pathway and Inhibits H9c2 Cardiomyocyte Injury Induced by Hypoxia-Reoxygenation
}

\author{
Ping Yang, ${ }^{a}$ Yuping Zhou, ${ }^{* b}$ Qing Xia, ${ }^{a}$ Lipeng Yao, ${ }^{a}$ and Xiuchun Chang ${ }^{a}$ \\ ${ }^{a}$ Ningbo College of Health Sciences; Ningbo 315100, China: and ${ }^{b}$ The Affiliated Hospital of Medical School of Ningbo \\ University; Ningbo 315020, China.
}

Received November 1, 2018; accepted January 20, 2019; advance publication released online March 13, 2019

\begin{abstract}
Astragaloside IV (AS-IV) is one of the main pharmacologically active compounds found in Astragalus membranaceus. AS-IV has protective effects against ischemia-reperfusion injury (IRI), but its mechanism of action has not yet been determined. This study aims to investigate the effect of AS-IV on IRI and its effect on the phosphadylinositol 3-kinase (PI3K)/Akt/heme oxygenase (HO-1) signaling pathway through in vitro experiments. Firstly, a cell culture model of myocardiocyte hypoxia-reoxygenation (H/R) injury was replicated. After AS-IV treatment, cell viability, reactive oxygen species (ROS) levels, as well as the content or activity of the cellular factors lactate dehydrogenase (LDH), superoxide dismutase (SOD), malondialdehyde (MDA), interleukin 6 (IL-6), tumor necrosis factor alpha (TNF- $\alpha$ ), were measured to evaluate the effect of treatment with AS-IV. The effect of AS-IV on HO-1 protein expression and nuclear factor E2-related factor 2 (Nrf2) and Bach1 protein expression was determined by Western blotting. Finally, a reversal of the effect of AS-IV treatment was observed following co-incubation with a PI3K inhibitor. Our results show that AS-IV has good protective effect on $H / R$ injury and has anti-oxidative stress and anti-inflammatory effects. It can regulate the expression of $\mathrm{Nrf} 2$ and Bach1 proteins in the nucleus and promote the expression of HO-1 protein, while a PI3K inhibitor can partially reverse the above effects. This study suggests that the PI3K/Akt/HO-1 signaling pathway may be a key signaling pathway for the anti-IRI effect of AS-IV.
\end{abstract}

Key words astragaloside IV; H9c2 cardiomyocyte; hypoxia-reoxygenation injury; heme oxygenase (HO-1); phosphadylinositol 3-kinase-Akt pathway

\section{INTRODUCTION}

The traditional Chinese medicine consisting of the root of Astragalus membranaceus BUNGE has been widely used in the treatment of various diseases for several thousands of years. Astragaloside-IV (AS-IV) is one of the main pharmacologically active substances of $A$. membranaceus Bunge. Studies have confirmed that AS-IV has protective effect sagainst cardiovascular disease, ischemic encephalopathy, diabetes, liver fibrosis, and other diseases. Its mechanism of action involves antiinflammatory, anti-oxidative, anti-fibrotic, and immunoregulatory actions. ${ }^{1)}$ In recent years, in vitro and in vivo studies have shown that AS-IV is protective against myocardial ischemiareperfusion injury (IRI) ${ }^{2,3)}$ and its mechanism of action is mainly related to anti-oxidative stress and anti-inflammatory pathways. ${ }^{4-6)}$ However, the specific target molecules and signaling pathways involved still await further study. Heme oxygenase (HO-1) is recognized as an important anti-oxidative stress and tissue protective enzyme that produces $\mathrm{CO}$ and biliverdin, and can also induce multiple anti-oxidative and anti-inflammatory signaling pathways, which significantly reduce cellular damage and protect organ function. ${ }^{7)}$ As a novel protective factor in myocardiocytes, HO-1 plays an important role in mitigating damage due to myocardial IRI through the anti-inflammatory, anti-oxidative stress, anti-apoptotic, and anti-arrhythmic effects of its products. ${ }^{8)}$ In this study, we further studied the mechanism through which AS-IV induces HO-1 expression and its related signaling pathways on an in vitro model of myocardial IRI.

\section{MATERIALS AND METHODS}

Main Materials The H9c2 rat cardiomyocyte cell line was purchased from The Institute of Life Sciences cell bank (Shanghai, China). AS-IV (Lot No. H-013-170614), purity 99.06\%, was purchased from Herbpurify Co., Ltd. (Chengdu, China). and its molecular structure is shown in Fig. 1. AS-IV $(5 \mathrm{~g})$ was stored at the Institute of Biochemistry and Molecular Biology of Ningbo University to permit future reference and verification.

Dulbecco's modefied Eagle's medium (DMEM) was purchased from Gibco Co. (Grand Island, NY, U.S.A.). Fetal bovine serum (FBS) was purchased from Hyclone Co. (Logan, UT, U.S.A.). Cell Counting Kit-8 (CCK-8) was purchased from

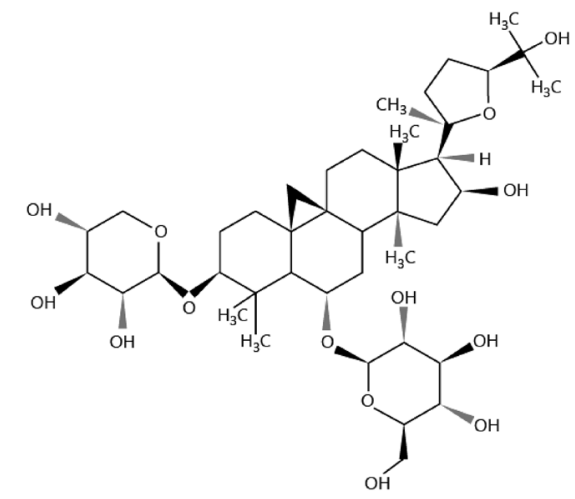

Fig. 1. Chemical Structure of AS-IV

Molecular formula, $\mathrm{C}_{41} \mathrm{H}_{68} \mathrm{O}_{14}$, molecular weight, $784.9702 \mathrm{~g} / \mathrm{mol}$. 
Lianke Biology Technology Co., Ltd. (Hangzhou, China). Reactive oxygen species (ROS), lactate dehydrogenase (LDH), superoxide dismutase (SOD), and malondialdehyde (MDA) kits were purchased from Institute of Bioengineering (Nanjing, China). Tumor necrosis factor alpha (TNF- $\alpha$ ) and interleukin-6 (IL-6) enzyme-linked immunosorbent assay (ELISA) kits were purchased from USCN Co., Ltd. (Wuhan, China). Cell lysis buffer for Western and nuclear protein extraction kit, BCA protein quantification kit, and ECL luminescence kit for Western blotting were purchased from Beyotime Biotechnology Co. (Shanghai, China). Phosphadylinositol 3-kinase (PI3K), p-Akt, Akt, HO-1, nuclear factor E2-related factor 2 (Nrf2), Bach1, glyceraldehyde-3-phosphate dehydrogenase (GAPDH), and HistoneH3 rabbit anti-rat polyclonal antibodies were purchased from Proteintech Co. (Shanghai, China). Cobalt protoporphyrin (CoPP, HO-lagonist) and zinc(II) protoporphyrin IX complex (ZnPP, HO-1 inhibitor) were purchased from Sigma-Aldrich Co. (St. Louis, MO, U.S.A.), PI3K inhibitor LY294002 was purchased from Selleck Co. (Houston, TX, U.S.A.).

Cell Culture and Hypoxia-Reoxygenation (H/R) Model Replication H9c2 cardiomyocytes were seeded in DMEM containing $10 \% \mathrm{FBS}$, placed in a constant temperature incubator at $37^{\circ} \mathrm{C}$ under $5 \% \mathrm{CO}_{2}$. Cells were subcultured based on their density and logarithmic growth phase (approximately $80 \%$ confluent) cells were used for experiments. After optimizing experimental conditions, the H9c2 H/R model was determined: the cells were cultured for $24 \mathrm{~h}$, then cultured for $12 \mathrm{~h}$ under hypoxia and hypoglycemia, followed by reoxygenation in media containing optimal glucose for $8 \mathrm{~h}$.

Toxicity of ASIV on Normal H9c2 Cells H9c2 cells in logarithmic growth phase were divided into the control group and four AS-IV treatment groups $(0.1,1,10,100 \mu \mathrm{M})$. After incubating for $20 \mathrm{~h}$, cell viability was measured using CCK-8. Cell viability $(\%)=($ test well OD - blank well OD $) /($ control well OD - blank well OD) $\times 100$.

Determination of AS-IV Effective Concentration H9c2 cells in logarithmic growth phase were divided into the control group, H/R model group, and four AS-IV treatment groups $(0.1,1,10,100 \mu \mathrm{M})$. During H/R model establishment, the cells were incubated in the presence of AS-IV for $20 \mathrm{~h}$, cell viability was measured using $\mathrm{CCK}-8$, and the LDH content of the cell supernatant was measured using the LDH kit.

Cell Grouping and Treatment Methods Logarithmic growth phase cells were randomly divided into the following groups: control group (cultured under normal conditions for $20 \mathrm{~h}$ ), H/R model group (hypoxic for $12 \mathrm{~h}$ followed by reoxygenation for $8 \mathrm{~h}), \mathrm{H} / \mathrm{R}+\mathrm{AS}-\mathrm{IV}$ group $(100 \mu \mathrm{M}$ AS-IV administered for $20 \mathrm{~h}$ during $\mathrm{H} / \mathrm{R}$ model establishment), $\mathrm{H} / \mathrm{R}+\mathrm{AS}-\mathrm{IV}+\mathrm{ZnPP}$ group $(100 \mu \mathrm{M}$ AS-IV and ZnPP administered for $20 \mathrm{~h}$ during $\mathrm{H} / \mathrm{R}$ model establishment), and $\mathrm{H} / \mathrm{R}+\mathrm{CoPP}$ group (positive control group, CoPP treatment for $20 \mathrm{~h}$ during $\mathrm{H} / \mathrm{R}$ model establishment). After treatment, the cell supernatant was collected and the concentrations or activities of SOD, MDA, IL- 6 , and TNF- $\alpha$ were measured. Three replicate wells were set up for each group and each experiment was performed in triplicate.

Determination of SOD and MDA Activities and IL-6 and TNF- $\alpha$ Content SOD activity was measured using the -hydroxylamine method, while the MDA activity was measured using the thiobarbituric acid method. The contents of
IL-6 and TNF- $\alpha$ were measured by ELISA. All measurements were performed according to kit instructions.

Determination of Intracellular ROS ROS levels were determined using 2,7-dichlorodihydrofluorescein diacetate (DCFH-DA) staining. Cell were seeded in 6-well plates, treated with AS-IV for $20 \mathrm{~h}$, Cells were stained with DCFH-DA $(10 \mu \mathrm{M})$ in a staining buffer for $30 \mathrm{~min}$ at $37^{\circ} \mathrm{C}$. Then, random cells were photographed under an inverted fluorescence microscope and five images were selected for each group, The intensity of fluorescence was determined and the mean fluorescence intensity (MFI) was determined.

Treatment with PI3K Inhibitor LY294002 Logarithmic growth phase cells were randomly divided into the following groups: control group (culture under normal conditions for $20 \mathrm{~h}$ ), H/R model group, H/R + ASIV group, and H/R + ASIV + LY294002 group $(20 \mu \mathrm{M}$ LY294002 pretreatment for $2 \mathrm{~h}$, followed by $100 \mu \mathrm{M}$ ASIV for $20 \mathrm{~h}$ ). At the end of the treatment, cells were collected for measurement of PI3K/Akt/HO-1 signaling pathway protein expression. Three replicate wells were set up for each group and the experiment was repeated in triplicate.

Western Blot Cells were collected and total protein (to assay PI3K, p-Akt, Akt and HO-1 content) as well as nuclear protein (to assay $\mathrm{Nrf} 2$ and Bach1 content) were extracted. Protein concentration was determined using the BCA method. Thirty micrograms of protein were loaded from each well and separated via gel electrophoresis on $12 \%$ polyacrylamide gel. The separated proteins were transferred onto polyvinylidene difluoride (PVDF) membranes. A blocking solution containing $5 \%$ skim milk was incubated with the membranes on a shaker at room temperature for $1 \mathrm{~h}$, followed by three TBST washes. Rabbit anti-rat PI3K, p-Akt, Akt, HO-1, Nrf2, and Bach1 polyclonal antibodies were added to the respective reaction and allowed to incubate overnight at $4^{\circ} \mathrm{C}$. The membrane was washed three times with TBST on the following day, then goat anti-rabbit immunoglobulin $\mathrm{G}(\mathrm{IgG})$ secondary antibody was added and incubated at room temperature for $1 \mathrm{~h}$. The membrane was washed 3 times with TBST. The ECL kit instructions were followed to expose the proteins of interest and the image was analyzed using Image J. The relative expression of each protein of interest was normalized using either GAPDH (PI3K, Akt, HO-1) or HistoneH3 (Nrf2, Bach1).

Statistical Analysis The data were analyzed and processed using SPSS19.0. Data were expressed as mean \pm standard deviation (S.D.) $(x \pm s)$. One-way ANOVA was used for comparison between groups. The Least-Significant Difference method was used for further comparison between the two groups. $p<0.05$ indicates statistically significant differences.

\section{RESULTS}

AS-IV Reduces Cytotoxicity to H9c2 Cells Induced by H/R Injury The CCK-8 assay demonstrates cell viability. The range of concentrations of AS-IV used in this experiment $(0.1-100 \mu \mathrm{M})$ have no significant effect on the viability of normal cultured H9c2 cells $(p>0.05)$ (Fig. 2A). Compared to the control group, the viability of the $H / R$ group decreased significantly $(p<0.01)$. Compared to the $\mathrm{H} / \mathrm{R}$ group, the AS-IV 10 and $100 \mu \mathrm{M}$ treatment groups showed significantly increased cell viability $(p<0.01)$, while the AS-IV 0.1 and $1 \mu \mathrm{M}$ treatment groups showed no significant effect (Fig. 
A

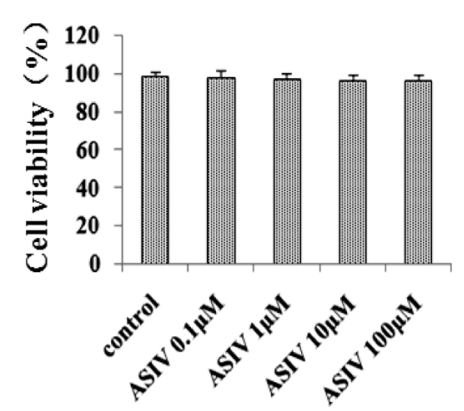

B

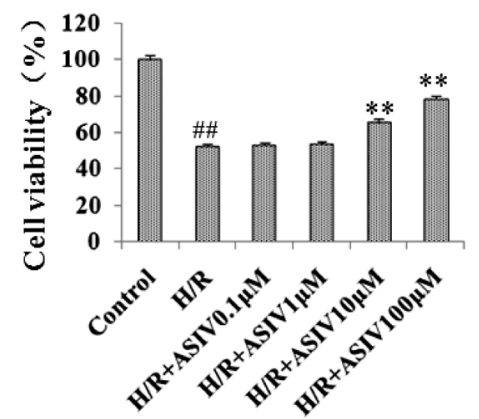

C

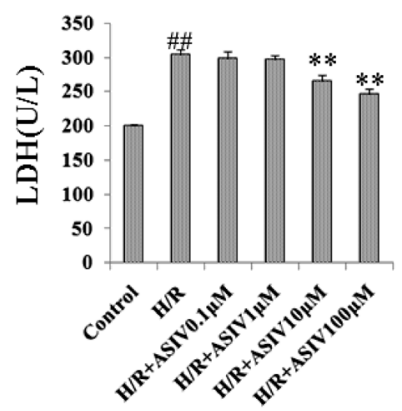

Fig. 2. AS-IV Attenuates H9c2 Cytotoxicity Induced by H/R Injury

(A) H9c2 cells were treated with AS-IV for $20 \mathrm{~h}$, and cell viability was measured using CCK-8. (B) Pre-I/R injury for $8 \mathrm{~h}$ and incubation with AS-IV for $12 \mathrm{~h}$, the cell viability was measured using CCK-8. (C) Pre-I/R injury for $8 \mathrm{~h}$ and incubation with AS-IV for $12 \mathrm{~h}$, the content or activity of the cellular LDH was measured. All experiments were repeated at least three times Data are presented as mean \pm S.D. ${ }^{\#} p<0.01$ vs. control group, ${ }^{*} * p<0.05$ vs. H/R group. $n=3$.
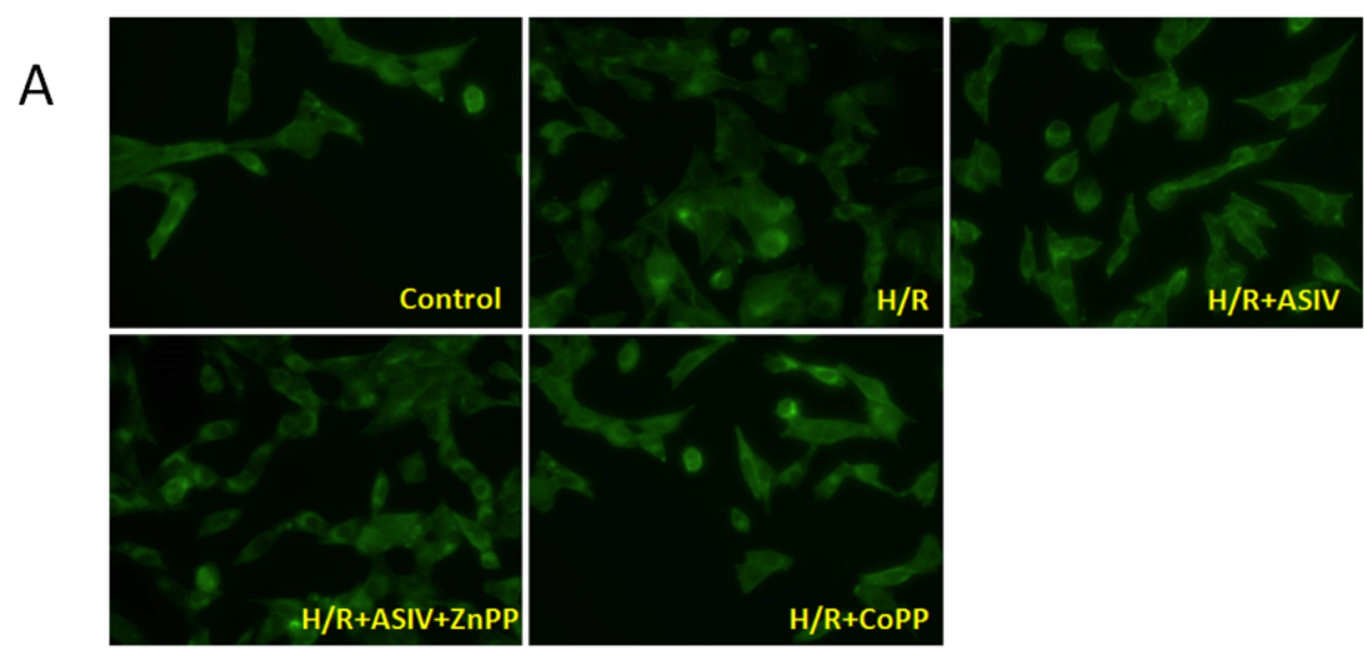

B

C
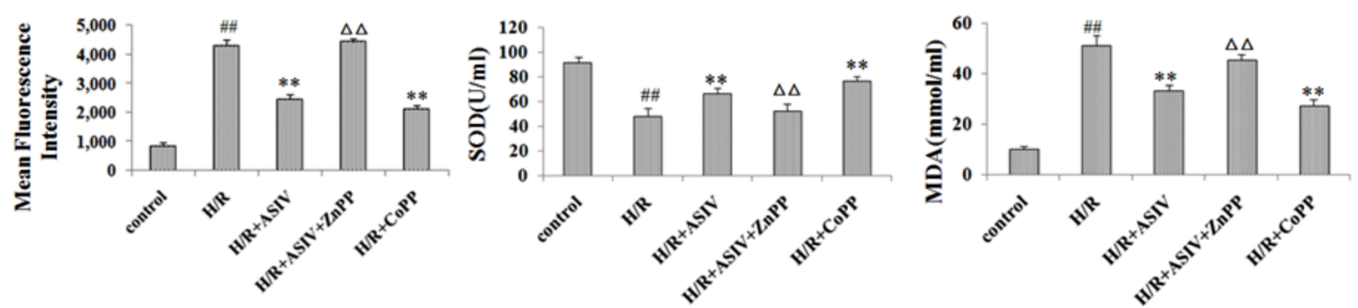

Fig. 3. AS-IV Attenuates Oxidative Damage in H9c2 Cells Induced by H/R Injury

(A, B) ROS levels in H9c2 cells were determined using DCFH-DA staining. (C) SOD activity was measured in the lysed cell supernatant; (D) MDA activity was measured in the lysed cell supernatant. Data are presented as mean \pm S.D. All experiments were repeated at least three times. ${ }^{\#} p<0.01 v s$. control group, $* * p<0.05 v s$. H/R group, ${ }^{\Delta \triangle} p<0.05 v s$. H/R + ASIV group. $n=3$. (Color figure can be accessed in the online version.)

2B). The LDH assay indicates that LDH activity in the H/R group was significantly higher than that in the control group $(p<0.01)$. Compared with the $\mathrm{H} / \mathrm{R}$ group, treatment with 10 and $100 \mu \mathrm{M}$ AS-IV significantly decreased the LDH activity level $(p<0.01)$. However, AS-IV administered at 0.1 and $1 \mu \mathrm{M}$ had no significant effect on LDH activity $(p>0.05)$ (Fig. $2 C)$. These results indicate that the viability of $H / R$ model cells is significantly decreased, and that $H / R$ injury-induced cytotoxicity is significantly increased. AS-IV treatment at concentrations of 10 and $100 \mu \mathrm{M}$ significantly increases cell viability and reduces cytotoxicity. As results using $100 \mu \mathrm{M}$ was superior to those obtained with $10 \mu \mathrm{M}$ AS-IV, subsequent experiments were performed with AS-IV100 $\mu \mathrm{M}$.
AS-IV Alleviates Oxidative Damage of H9c2 Cells Induced by $\mathbf{H} / \mathbf{R}$ Injury Experimental results show that when compared to the control group, ROS level and MDA activity increased significantly in the H/R group, while SOD activity decreased significantly $(p<0.01)$. Compared with the $H / R$ group, ROS level and MDA activity decreased significantly in the AS-IV and CoPP treatment groups, while SOD activity increased significantly $(p<0.01)$. Compared with the AS-IV group, ROS level and MDA activity increased significantly in the ASIV + ZnPP treatment group, while SOD activity decreased significantly $(p<0.01)$ (Figs. 3A, B). These findings indicate that AS-IV and CoPP have significant anti-oxidative stress effects, and ZnPP can partially reverse the anti-oxida- 

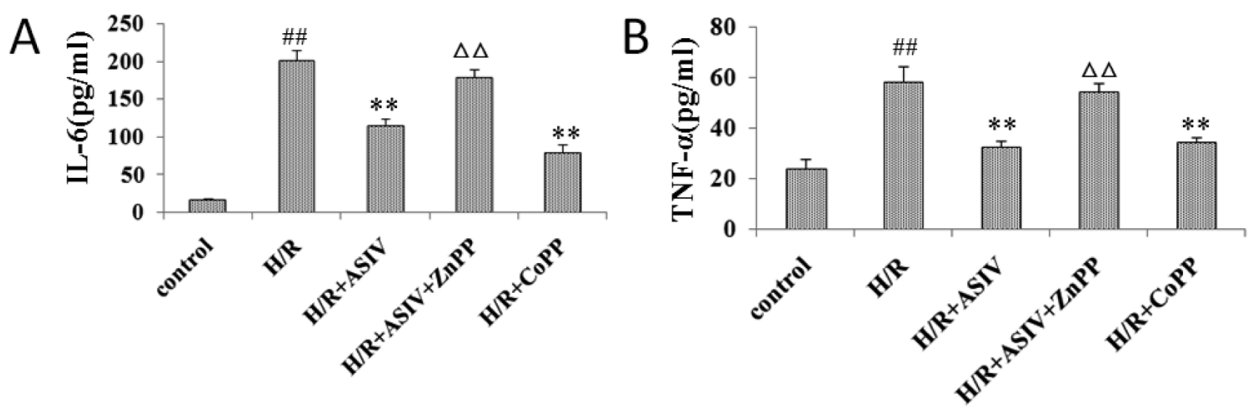

Fig. 4. AS-IV Attenuates H/R Injury-Induced Inflammatory Response in H9c2 Cells

(A) ELISA of secreted IL-6 in lysed cell supernatant; (B) ELISA of secreted TNF- $\alpha$ in lysed cell supernatant. Data are presented as mean \pm S.D. All experiments were repeated at least three times. ${ }^{\# \#} p<0.01$ vs. control group, ${ }^{* *} p<0.05 v s$. H/R group, ${ }^{\triangle} p<0.05 v s . \mathrm{H} / \mathrm{R}+\mathrm{ASIV}$ group. $n=3$.
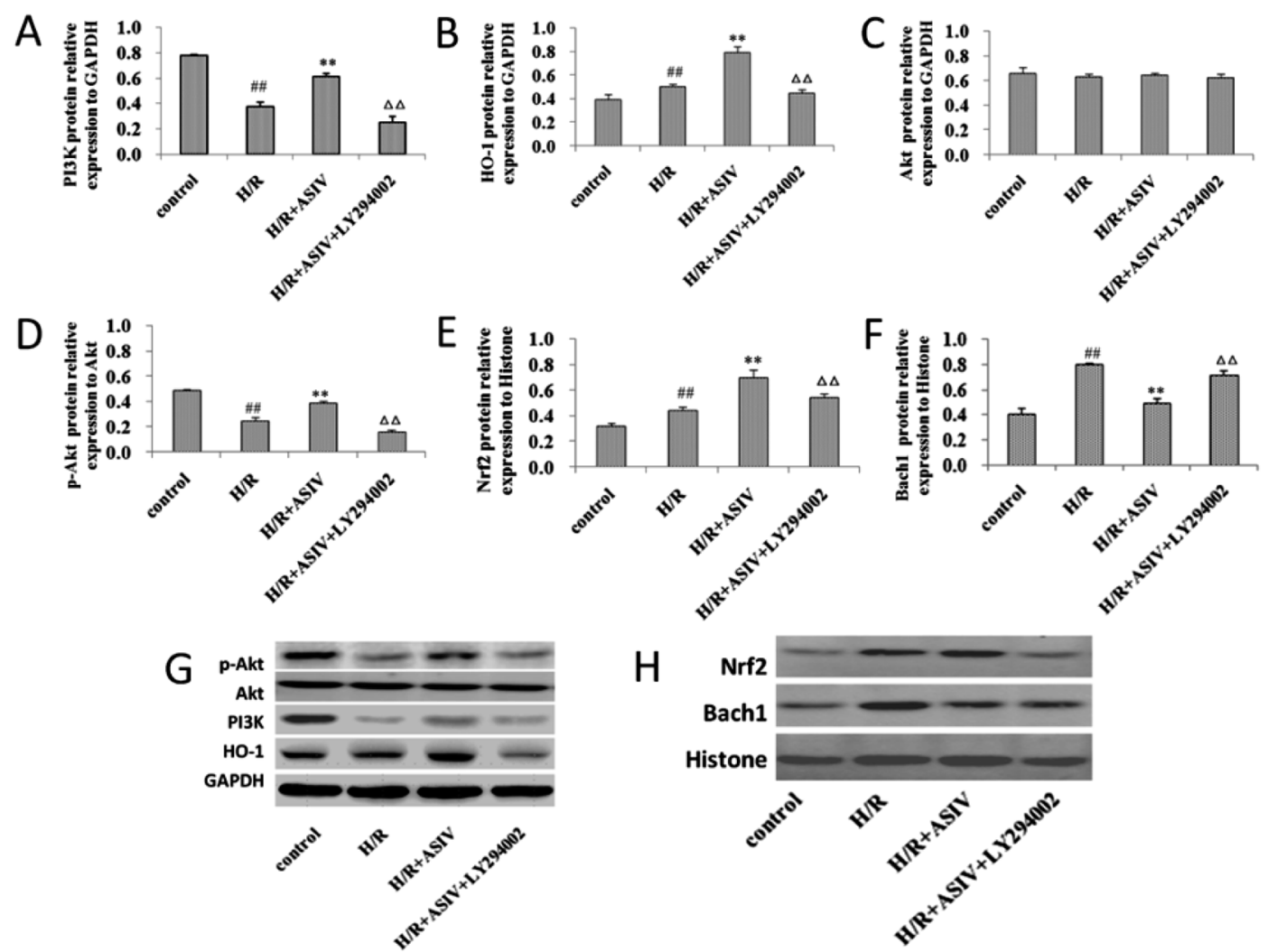

Fig. 5. Effect of AS-IV on Activation of PI3K/Akt/HO-1 Signaling Pathway

A, B, and G show the expression of PI3K and HO-1 protein in the total protein extract and their semi-quantitative analysis when normalized to GAPDH, C, D, and G show the the expression of Akt protein and and its level of phosphorylation. E, F, and $\mathrm{H}$ show the expression of Nrf2 and Bach1 and their semi-quantitative expression analysis when normalized to histone. LY294002, a specific inhibitor of PI3K, was administered concurrently with AS-IV for $20 \mathrm{~h}$ at a concentration of $20 \mu \mathrm{M}$. All experiments were repeated at least three times. Data are presented as mean \pm S.D. ${ }^{\# \#} p<0.01 v s$. control group, ${ }^{* *} p<0.05 v s$. H/R group, ${ }^{\Delta \Delta} p<0.05 v s$. H/R + ASIV group. $n=3$.

tive stress effects of AS-IV.

AS-IV Reduces the Inflammatory Response of H9c2 Cells Induced by $\mathbf{H} / \mathbf{R}$ Injury Experimental results show that compared with the control group, the levels of IL-6 and TNF- $\alpha$ secreted by cells in the H/R group were significantly increased $(p<0.01)$. Compared to the $\mathrm{H} / \mathrm{R}$ group, the cells in the AS-IV and CoPP treatment groups secreted significantly less IL-6 and TNF- $\alpha(p<0.01)$. Compared with the AS-IV group, IL-6 and TNF- $\alpha$ secretion increased significantly in the AS-IV $+\mathrm{ZnPP}$ treatment group $(p<0.01)$ (Figs. 4A, B). This indicates that AS-IV and CoPP have significant antiinflammatory effects and that ZnPP can partially reverse the anti-inflammatory effects of AS-IV.

Effect of AS-IV on Activation of PI3K/Akt/HO-1 Signaling Pathway Western blotting showed that the expression of
PI3K and p-Akt in the H/R group was significantly lower than that in the control group $(p<0.01)$. Compared with the $\mathrm{H} / \mathrm{R}$ group, the expression of PI3K and p-Akt in the AS-IV group increased significantly $(p<0.01)$. Compared with the AS-IV group, PI3K and p-Akt expression in the PI3K-specific inhibitor LY294002 group decreased significantly $(p<0.01)$ (Figs. $5 \mathrm{~A}, \mathrm{D}, \mathrm{G})$. Compared with the control group, the expression of HO-1 in the H/R group increased significantly increased $(p<0.01)$. Compared with the $\mathrm{H} / \mathrm{R}$ group, the expression of HO-1 in the AS-IV group increased significantly $(p<0.01)$. In comparison, the expression of HO-1 in the LY294002 group decreased significantly $(p<0.01)$ (Figs. 5B, G). The results suggest that AS-IV promotes HO-1 protein expression, and PI3K-specific inhibitors partially reverse this effect. In addition, AS-IV had no influence to the basal expression of PI3K, 
p-Akt, HO-1, Nrf2 and Bach1 $(p>0.05)$ (Fig. S1 available online).

Western blotting also showed that the expression of Nrf2 in the $\mathrm{H} / \mathrm{R}$ group was significantly higher than that in the control group $(p<0.01)$. Compared with the H/R group, the expression of Nrf2 in the AS-IV group increased significantly $(p<0.01)$. Compared with the AS-IV group, the expression of Nrf2 in the LY294002 group decreased significantly $(p<0.01)$ (Figs. 5E, H). Compared with the control group, the expression of Bach1 increased significantly in the H/R group $(p<0.01)$. Compared to the $\mathrm{H} / \mathrm{R}$ group, the expression of Bach1 decreased significantly in the AS-IV group $(p<0.01)$. Compared with the AS-IV group, the expression of Bach1 increased significantly in the LY294002 group $(p<0.01)$ (Figs. 5F, H). The results suggest that AS-IV can significantly increase nuclear Nrf2 expression, while decreasing nuclear Bach1 expression, and PI3K specific inhibitors can partially reverse this effect.

\section{DISCUSSION}

Reducing myocardial IRI has been a primary focus of researchers and clinicians. ${ }^{9)}$ It is generally believed that the occurrence and development of IRI is a due to a cascade of multi-factor interactions, which are mutually causal and mutually influential, and ultimately lead to the apoptosis and death of cardiomyocytes. Free radical damage and inflammatory responses are believed to play key roles in myocardial IRI. ${ }^{10)}$ In recent years, the role of HO-1 in myocardial IRI has received a great deal of attention. Numerous studies have shown that HO-1 and its metabolites are widely involved in anti-oxidative stress, anti-inflammatory damage, anti-cell proliferation, anti-apoptosis, inhibition of platelet aggregation, regulation of vascular tone, and intracellular signaling and play a very important role in cardiovascular diseases. ${ }^{8}$ HO-1 appears to be particularly closely related to myocardial IRI, as the expression of HO-1 is significantly increased in the early stage of myocardial IRI. ${ }^{11)}$ Over-expression of the HO-1 gene in cardiomyocytes or increased myocardial HO-1 expression via gene therapy or drug therapy can significantly reduce the infarct surface area due to ischemia during IRI and significantly improve myocardial function. ${ }^{12-14)}$ Mice deficient in the HO-1 gene showed more severe IRI. ${ }^{15)}$ In this study, by replicating the myocardial H/R cell culture model, we were able to separately administer HO-1 agonists and inhibitors. The study also confirmed that HO-1 can significantly inhibit the expression of inflammatory factors and increase the level of anti-oxidants, indicating that $\mathrm{HO}-1$ is protective against myocardial $\mathrm{H} / \mathrm{R}$ injury via protection against oxidative stress and inhibition of inflammation.

The above results suggest that by highly expressing HO-1 levels, myocardial IRI can be attenuated to some extent. Both in vitro and in vivo experiments have confirmed that AS-IV has significant protective effects against myocardial IRI, but its mechanism of action needs to be further explored. In this study, the mechanism of action of AS-IV was further studied using an in vitro myocardial $\mathrm{H} / \mathrm{R}$ model. The results show that after treatment with AS-IV, the expression of HO-1 is significantly increased, the secretion of cytokines is significantly reduced, and the anti-oxidative stress capacity of cells is enhanced. A HO-1 inhibitor partially reversed the above effects, suggesting that induction of HO-1 was the source of the above changes. The expression of HO-1 is one of the mechanisms of action by which AS-IV protects against myocardial H/R injury. But what is the signaling pathway through which AS-IV induces HO-1?

Studies have shown that the regulation of HO-1 gene expression occurs mainly at the transcriptional level. Nuclear factor E2-related factor 2 (Nrf2) is an important transcriptional regulator of anti-oxidative stress. ${ }^{16)}$ When cardiomyocytes are under oxidative stress, Nrf2 is rapidly phosphorylated, dissociates, activates, and translocates to the nucleus, where it binds to the anti-oxidative stress response element (ARE) to promote the expression of anti-oxidant proteins such as HO-1 and SODto combat oxidative stress caused by ischemia and hypoxia. ${ }^{17)}$ Tanaka et al. found that deletion of the Nrf2 gene results in a significant decrease in the expression of various baseline and inducible anti-oxidant genes, including $\mathrm{HO}-1$, and an overall increase in oxidative damage. ${ }^{18)}$ On the other hand, gene regulation of $\mathrm{HO}-1$ is also negatively regulated by some Nrf2 repressors, such as BTB-CNC allogeneic 1 (BTB and $\mathrm{CNC}$ homology 1, Bach1), and deletion of the Bach1 gene can lead to increased expression of HO-1. ${ }^{19)}$ The mechanism by which Bach1 and Nrf2 antagonize the expression of antioxidant genes may be related to the competitive binding of ARE sequences in the nucleus. Oxidative stress phosphorylates Bach1 tyrosine 486, allowing for rapid nuclear exit and for Nrf2 to bind to available AREs, resulting in the production of anti-oxidant enzymes. ${ }^{20}$ The above experimental results suggest that the dynamic equilibrium relationship between Nrf2 and Bach1 in the nucleus affects the transcription of antioxidant enzymes such as HO-1.

Phosphadylinositol 3-kinase (PI3K) is an intracellular phosphatidylinositol kinase that is a second messenger located on the plasma membrane Akt is a key downstream gene in the PI3K/Akt pathway. Akt induces phosphorylation of serine and threonine residues and activates or inhibits downstream target gene expression. ${ }^{21}$ ) The PI3K/Akt signaling pathway is essential for heart and vascular health. ${ }^{22)}$ As an important part of the body's protective mechanism, the PI3K/Akt pathway plays a major role in controlling cell growth, survival, proliferation, and migration, and improves overall function in IRI through activating $\mathrm{Akt}^{23,24)} \mathrm{PI} 3 \mathrm{~K}$ may affect the expression of HO-1 by regulating the balance of Nrf2 and Bach1. Sun et al. showed that inhibition of the PI3K/Akt pathway significantly reduces $\mathrm{HO}-1$ protein expression, thereby attenuating the protective effects of HO-1 on cardiomyocytes. ${ }^{25)}$ Joung et al. stimulated HepG2 cells with capsaicin to activate the PI3K/Akt pathway, increasing the nuclear translocation of Nrf2 and the transcriptional activity of AREs, and thereby increasing the expression of HO-1 in cells. ${ }^{26)}$ Other studies have shown that chlorophyllin induces nuclear translocation of Nrf2 via activation of the PI3K pathway, up-regulating HO-1 expression in human umbilical vein endothelial cells in a time- and dose-dependent manner. ${ }^{27)}$ These findings strongly confirm that PI3K/Akt upregulates the Nrf2-ARE pathway and mediates HO-1 expression. There are fewer reports on whether PI3K-mediated HO-1 expression is simultaneously achieved by down-regulating Bach1 activity. In an experiment on Jurkat tumor cells, the PI3K signaling pathway was shown to activate Nrf2 and inhibit Bach1 activity, thereby regulating HO-1 expression. ${ }^{28)}$ 
However, there are only a few studies on whether PI3K/Akt signaling pathway regulates HO-1 in myocardial IRI. A recently study showed that, a Chinese herbal medicine extract (TFCC) protects against myocardial injury and enhances cellular antioxidant defense capacity by inducing the phosphorylation of Akt, which subsequently activates the $\mathrm{Nrf} 2 / \mathrm{HO}-1$ signaling pathway. ${ }^{29)}$ In addition, two studies showed that AS-IV has protective effects on the cardiovascular system by regulating the PI3K/Akt signaling pathway. ${ }^{30,31)}$ However, whether AS-IV further induces HO-1 expression by regulating the PI3K/Akt signaling pathway and its downstream effectors remains unclear. The results of this study show that the effect of AS-IV on myocardial H/R injury can significantly increase the expression of PI3K and p-Akt in cells, thereby increasing the nuclear expression of Nrf2 and decreasing the nuclear expression of Bach1, and further increased the expression of HO-1 protein, while a PI3K-specific inhibitor can partially reverse the above effects. These results suggest that AS-IV may affect the nuclear translocation of Nrf2 and Bach1 by Activating the PI3K/Akt signaling pathway thereby regulating the expression of HO-1. Therefore, this study confirms that the $\mathrm{PI} 3 \mathrm{~K} / \mathrm{Akt} / \mathrm{HO}-1$ signaling pathway is a key signaling pathway for the anti-myocardial H/R injury of AS-IV.

Acknowledgments This study was supported by the Natural Science Foundation of Ningbo municipality (No. 2017A610260).

Conflict of Interest The authors declare no conflict of interest.

Supplementary Materials The online version of this article contains supplementary materials.

\section{REFERENCES}

1) Li L, Hou X, Xu R, Liu C, Tu M. Research review on the pharmacological effects of astragaloside IV. Fundam. Clin. Pharmacol., 31, 17-36 (2017).

2) Zhang WD, Chen H, Zhang C, Liu RH, Li HL, Chen HZ. Astragaloside IV from Astragalus membranaceus shows cardioprotection during myocardial ischemia in vivo and in vitro. Planta Med., $\mathbf{7 2}$, 4-8 (2006).

3) Zheng Q, Zhu JZ, Bao XY, Zhu PC, Tong Q, Huang YY, Zhang QH, Zhang KJ, Zheng GQ, Wang Y. A preclinical systematic review and meta-analysis of astragaloside IV for myocardial ischemia/reperfusion injury. Front. Physiol., 9, 795 (2018).

4) Lu M, Tang F, Zhang J, Luan A, Mei M, Xu C, Zhang S, Wang H, Maslov LN. Astragaloside IV attenuates injury caused by myocardial ischemia/reperfusion in rats via regulation of toll-like receptor 4/nuclear factor- $\kappa \mathrm{B}$ signaling pathway. Phytother. Res., 29, 599-606 (2015).

5) Huang H, Lai S, Wan Q, Qi W, Liu J. Astragaloside IV protects cardiomyocytes from anoxia/reoxygenation injury by upregulating the expression of Hes1 protein. Can. J. Physiol. Pharmacol., 94, 542-553 (2016)

6) Wang SG, Xu Y, Chen XH. Effects of astragaloside IV on hypoxiareoxygenation injury of $\mathrm{H} 9 \mathrm{c} 2$ cardiomyocytes. Pharmacology and Clinics of Chinese Materia Medica, 30, 45-48 (2014).

7) Singh N, Ahmad Z, Baid N, Kumar A. Host heme oxygenase-1: friend or foe in tackling pathogens? IUBMB Life, 70, 869-880 (2018).

8) Otterbein LE, Foresti R, Motterlini R. Heme oxygenase-1 and carbon monoxide in the heart: the balancing Act between danger signaling and pro-survival. Circ. Res., 118, 1940-1959 (2016).

9) Ibáñez B, Heusch G, Ovize M, Van de Werf F. Evolving therapies for myocardial ischemia/reperfusion injury. J. Am. Coll. Cardiol., 65, 1454-1471 (2015)

10) Cadenas S. ROS and redox signaling in myocardial ischemia-reperfusion injury and cardioprotection. Free Radic. Biol. Med., 117, 76-89 (2018).

11) Maulik N, Sharma HS, Das DK. Induction of the heme oxygenase gene-expression during the reperfusion of ischemic rat myocardium. J. Mol. Cell. Cardiol., 28, 1261-1270 (1996).

12) Yet SF, Tian R, Layne MD, Wang ZY, Maemura K, Solovyeva M, Ith B, Melo LG, Zhang L, Ingwall JS, Dzau VJ, Lee ME, Perrella MA. Cardiac-specific expression of heme oxygenase-1 protects against ischemia and reperfusion injury in transgenic mice. Circ. Res., 89, 168-173 (2001).

13) Hinkel R, Lange P, Petersen B, Gottlieb E, Ng JK, Finger S, Horstkotte J, Lee S, Thormann M, Knorr M, El Aouni C, Boekstegers P, Reichart B, Wenzel P, Niemann H, Kupatt C. Heme oxygenase-1 gene therapy provides cardioprotection via control of post-ischemic inflammation: an experimental study in a pre-clinical pig model. $J$. Am. Coll. Cardiol., 66, 154-165 (2015).

14) Liu X, Simpson JA, Brunt KR, Ward CA, Hall SR, Kinobe RT, Barrette V, Tse MY, Pang SC, Pachori AS, Dzau VJ, Ogunyankin KO, Melo LG. Preemptive heme oxygenase-1 gene delivery reveals reduced mortality and preservation of left ventricular function 1 yr after acute myocardial infarction. Am. J. Physiol. Heart Circ. Physiol., 293, H48-H59 (2007).

15) Liu X, Wei J, Peng DH, Layne MD, Yet SF. Absence of heme oxygenase-1 exacerbates myocardial ischemia/reperfusion injury in diabetic mice. Diabetes, 54, 778-784 (2005).

16) Ndisang JF. Synergistic interaction between heme oxygenase (HO) and nuclear-factor E2-related factor-2 (Nrf2) against oxidative stress in cardiovascular related diseases. Curr. Pharm. Des., 23, 1465-1470 (2017)

17) Xue M, Momiji H, Rabbani N, Barker G, Bretschneider T, Shmygol A, Rand DA, Thornalley PJ. Frequency modulated translocational oscillations of Nrf2 mediate the antioxidant response element cytoprotective transcriptional response. Antioxid. Redox Signal., 23, 613-629 (2015)

18) Tanaka Y, Maher JM, Chen C, Klaassen CD. Hepatic ischemia-reperfusion induces renal heme oxygenase-1 via NF-E2-related factor 2 in rats and mice. Mol. Pharmacol., 71, 817-825 (2007).

19) Omura S, Suzuki H, Toyofuku M, Ozono R, Kohno N, Igarashi K. Effects of genetic ablation of bach1 upon smooth muscle cell proliferation and atherosclerosis after cuff injury. Genes Cells, 10, 277-285 (2005)

20) Baird L, Dinkova-Kostova AT. The cytoprotective role of the Keap1-Nrf2 pathway. Arch. Toxicol., 85, 241-272 (2011).

21) Jafari M, Ghadami E, Dadkhah T, Akhavan-Niaki H. PI3k/AKT signaling pathway: erythropoiesis and beyond. J. Cell. Physiol., 234, 2373-2385 (2019).

22) Abeyrathna P, Su Y. The critical role of Akt in cardiovascular function. Vascul. Pharmacol., 74, 38-48 (2015).

23) Zhao MM, Yang JY, Wang XB, Tang CS, Du JB, Jin HF. The PI3K/Akt pathway mediates the protection of $\mathrm{SO}(2)$ preconditioning against myocardial ischemia/reperfusion injury in rats. Acta Pharmacol. Sin., 34, 501-506 (2013).

24) Zhang SB, Liu TJ, Pu GH, Li BY, Gao XZ, Han XL. MicroRNA-374 exerts protective effects by inhibiting SP1 through activating the PI3K/Akt pathway in rat models of myocardial ischemia-reperfusion after sevoflurane preconditioning. Cell. Physiol. Biochem., 46, $1455-1470$ (2018).

25) Sun GB, Sun X, Wang M, Ye JX, Si JY, Xu HB, Meng XB, Qin M, Sun J, Wang HW, Sun XB. Oxidative stress suppression by luteolininduced heme oxygenase-1 expression. Toxicol. Appl. Pharmacol., 
265, 229-240 (2012).

26) Joung EJ, Li MH, Lee HG, Somparn N, Jung YS, Na HK, Kim SH, Cha YN, Surh YJ. Capsaicin induces heme oxygenase-1 expression in HepG2 cells via activation of PI3K-Nrf2 signaling: NAD(P)H: quinone oxidoreductase as a potential target. Antioxid. Redox Signal., 9, 2087-2098 (2007).

27) Zhang Y, Guan L, Wang X, Wen T, Xing J, Zhao J. Protection of chlorophyllin against oxidative damage by inducing $\mathrm{HO}-1$ and NQO1 expression mediated by PI3K/Akt and Nrf2. Free Radic. Res., 42, 362-371 (2008).

28) Sakamoto K, Iwasaki K, Sugiyama H, Tsuji Y. Role of the tumor suppressor PTEN in antioxidant responsive element-mediated transcription and associated histone modifications. Mol. Biol. Cell, 20,
1606-1617 (2009).

29) Zhang HJ, Chen RC, Sun GB, Yang LP, Zhu YD, Xu XD, Sun XB. Protective effects of total flavonoids from Clinopodium chinense (BENTH.) O. KTZE on myocardial injury in vivo and in vitro via regulation of Akt/Nrf2/HO-1 pathway. Phytomedicine, 40, 88-97 (2018).

30) Lin XP, Cui HJ, Yang AL, Luo JK, Tang T. Astragaloside IV improves vasodilatation function by regulating the PI3K/Akt/eNOS signaling pathway in rat aorta endothelial cells. J. Vasc. Res., $\mathbf{5 5}$ 169-176 (2018)

31) Jia Y, Zuo D, Li Z, Liu H, Dai Z, Cai J, Pang L, Wu Y. Astragaloside IV inhibits doxorubicin-induced cardiomyocyte apoptosis mediated by mitochondrial apoptotic pathway via activating the PI3K/Akt pathway. Chem. Pharm. Bull., 62, 45-53 (2014). 